\title{
Synthesis and Study of an Efficient Metal-Organic Framework Adsorbent (MIL-96(Al)) for Fluoride Removal from Water
}

\author{
Xingang Wang, ${ }^{1,2}$ Hui Zhu, ${ }^{1,2}$ Tongshuai Sun,, ${ }^{1,2}$ Yinbo Liu, ${ }^{1,2}$ Ting Han, ${ }^{1,2}$ Jingxian Lu,, \\ Hongliang Dai $\mathbb{D}^{1,2,3}$ and Linzhi Zhai $\mathbb{D}^{1,2}$ \\ ${ }^{1}$ School of Environmental and Chemical Engineering, Jiangsu University of Science and Technology, No. 2 Mengxi Road, \\ Zhenjiang 212018, China \\ ${ }^{2}$ Marine Equipment and Technology Institute, Jiangsu University of Science and Technology, Zhenjiang 212003, China \\ ${ }^{3}$ School of Energy and Environment, Southeast University, No. 2 Sipailou, Nanjing 210096, China
}

Correspondence should be addressed to Hongliang Dai; daihongliang@just.edu.cn and Linzhi Zhai; zhailinzhi@just.edu.cn

Received 25 April 2019; Revised 17 July 2019; Accepted 2 August 2019; Published 4 September 2019

Guest Editor: Fei Ke

Copyright (C) 2019 Xingang Wang et al. This is an open access article distributed under the Creative Commons Attribution License, which permits unrestricted use, distribution, and reproduction in any medium, provided the original work is properly cited.

Long-term consumption of drinking water that contains excessive amounts of fluoride can endanger human health; therefore, the preparation and application of a high-efficiency defluorination adsorbent for advanced purification of drinking water are of significant interest. This study presents a metal-organic framework adsorbent (MIL-96(Al)) with the granular structure of rice, which was generated by hydrothermal reaction. The specific surface area of MIL-96(Al) was $\sim 220 \mathrm{~m}^{2} \mathrm{~g}^{-1}$, and it showed a good thermal stability. Several experiments were conducted wherein conditions, including adsorbent dosage, initial concentration, $\mathrm{pH}$, and coexisting anions, were varied to understand the defluorination performance of the material. Results showed that $\mathrm{pH}$ (6-9) and coexisting anions had little effect on the removal efficiency of fluoride. The adsorption isotherm can be described by the Langmuir model, and the theoretical fluoride adsorption capacity of MIL-96(Al) was up to $42.19 \mathrm{mg} \mathrm{Fg}^{-1}$ at $298 \mathrm{~K}^{\text {, which is }}$ much higher than that of the commonly used activated alumina. The adsorption process of fluoride is endothermic and follows pseudo second-order kinetics. In addition, MIL-96(Al) was shown to still achieve $\sim 61.8 \%$ of the adsorption capacity after seven regenerations. This study shows that MIL-96(Al) is a good application prospect and could be widely used to remove fluoride from water.

\section{Introduction}

Fluoride is closely related to activities of human life and the metabolism of bone tissue. Excessive fluoride in natural water is predominantly caused by the discharge of fluoride-rich wastewater effluent or surface water washing of fluoridecontaining ore over long periods $[1,2]$. Excessive fluoride in the human body can mineralize calcium hydroxyphosphate to calcium fluorophosphate, which causes a significant change in the fluoride-phosphorus ratio resulting in spurs, periosteal hyperplasia, osteoporosis, osteosclerosis, and bone deformation [3, 4]. Long-term intake of high-fluoride food by children will result in enamel hypoplasia, tooth damage, and fluoride deposition, causing a series of deformities in the bones $[5,6]$. The World Health Organization (WHO) stipulates that the maximum fluoride concentration within drinking water should be $1.5 \mathrm{mg} \mathrm{L}^{-1}$ [7]; however, this is exceeded in some developing countries, especially in tropical countries. Therefore, it is generally recommended that the maximum concentration of fluoride in drinking water be $1.0 \mathrm{mg} \mathrm{L}^{-1}$ [8]. There are several methods that can be used to remove fluoride from water, such as precipitation, adsorption, electrocoagulation, ion exchange, reverse osmosis, and electrodialysis [9]. The equipment cost and energy consumption of the electrochemical methods are relatively large, the electrode is easy to passivate, and the selectivity of this method needs to be improved; the processing speed of the precipitation methods is slow, and the water quality of the effluent is not easy to reach the standard; the cost of the surface assimilation techniques needs to be reduced. Among 
these methods, the adsorption method is widely used for defluorination; however, there is still an urgent need for a low-energy consumption, simple, fast, efficient, and economical adsorbent [10].

Aluminum $(\mathrm{Al})$ is an abundant and inexpensive metal element and has a stronger bond strength with fluoride than many other adsorbents; therefore, activated alumina is the most commonly used metal adsorbent and is recognized by the WHO and the United States Environmental Protection Agency (USEPA) as the best fluoride removal adsorbent [11-13]. $\mathrm{Al}^{3+}$ also has excellent adsorption properties for other low-concentration contaminants [14]. Activated alumina has a large specific surface area; however, its adsorption capacity is only $0.8-2.0 \mathrm{mgg}^{-1}$ for fluoride, and it also requires adjustment of the $\mathrm{pH}$ of the reaction system, which increases the cost of water treatment [15]. For example, according to Dhillon et al.'s research, a Fe-Ca-Zr hybrid metal oxide nanomaterial has a large adsorption capacity of $250 \mathrm{mg} \mathrm{Fg}^{-1}$, but has a high $\mathrm{pH}$ requirement. [16]. In Venditti et al.'s study, industrial wastewater was treated with aluminum chloride containing Actifluo (Brenntag). Some pretreatment is also required, and the fluoride content in the treated wastewater is still relatively high [17]. In addition, coexisting anions in the water interfere with activated alumina, inhibiting the removal of fluoride from the water [18]. Of particular concern is that $\mathrm{Al}$ can leach into the water, which may cause harm to people's memory and intelligence and could even cause brain damage $[19,20]$. Powdered activated carbon also has a good fluoride removal performance; however, the fluoride removal process must be carried out under acidic conditions, increasing costs. To overcome these problems, a stable, convenient, and efficient Al-based defluorination adsorbent with a large adsorption capacity is required.

Metal-organic frameworks (MOFs) are crystalline porous materials with a periodic network structure composed of a porous metal center (metal ions or metal clusters) and a bridged organic ligand [21]. MOFs are also known as a coordination complex (coordination polymer) and have a high void ratio and a regular surface structure, which is different from general inorganic porous materials and organic complexes, and excellent chemical and thermal stability $[22,23]$. These characteristics mean that MOFs have many potential applications and are widely used in industries, for example, catalysis, sensors, gas adsorption, removal of hazardous materials, and potential drug delivery and biomedical applications [24-30]. As far as we know, the application of MOF due to poor water stability in water treatment is not much in the literature; for example, $\mathrm{AlFu}$ MOF was studied by Karmakar et al. Researches on the use of Al-based MOF or even MOF to adsorb fluoride from water are relatively rare $[31,32]$. This is probably because traditional MOFs are not suitable for use as defluorination adsorbents, and complex water quality conditions have a series of uncontrollable effects, where the combination of metal ions and fluorion results in a series of complex reactions [33]. Aluminum (Al) ions and fluorion complex in water to adsorbed fluoride and fluorion in water are mainly removed by means of physical adsorption, chemical adsorption, sweeping, and ion exchange of ligands. Due to the unique octahedral nature of the $\mathrm{Al}$ center [34], Al-MOF is used as a defluorination adsorbent, allowing $\mathrm{Al}$ ions to fully contact fluorion, resulting in improved fluoride removal.

Many previous studies have attempted to modify activated alumina and investigated several new adsorbents; however, the modifications resulted in no clear improvement in fluoride removal efficiency. In this study, a MOF (MIL-96(Al)), as a fluoride removal adsorbent, was synthesized and provides more active sites that react with fluoride, resulting in improved performance. The adsorption properties of MIL-96(Al) were explored under conditions varying by dosage, initial concentration, $\mathrm{pH}$, and coexisting anions. The adsorption isotherms, thermodynamics, kinetics, regeneration of material, and postregeneration performance were also investigated. A more effective and promising defluorination adsorbent was produced, and the results shed light on the adsorption mechanisms of various adsorbents for defluorination.

\section{Materials and Methods}

2.1. Materials. Aluminum nitrate nonahydrate $\left(\mathrm{Al}\left(\mathrm{NO}_{3}\right)_{3} \cdot 9 \mathrm{H}_{2} \mathrm{O}\right)$ and 1,3,5-benzentricarboxylate (1,3,5BTC or $\mathrm{H}_{3}$ BTC) were provided by Sinopharm Chemical Reagent Co., Ltd. (Shanghai, China). Sodium fluoride was provided by Tianjin Kaitong Chemical Reagent Co., Ltd. (Tianjin, China). Alizarin complexone (fluoride reagent, molecular formula: $\mathrm{C}_{19} \mathrm{H}_{15} \mathrm{NO}_{8}$ ) was provided by Tianjin Komiou Chemical Reagent Co., Ltd. (Tianjin, China). All of the chemical reagents used were of analytical grade and used as received without further purification.

2.2. Fabrication of MIL-96(Al). Synthesis of MIL-96(Al) was achieved by the reaction of $\mathrm{Al}^{3+}$ and a ligand at high temperature. $\mathrm{Al}\left(\mathrm{NO}_{3}\right)_{3} \cdot 9 \mathrm{H}_{2} \mathrm{O}(2.628 \mathrm{~g})$ and 1,3,5-BTC $(0.21 \mathrm{~g})$ were added to pure water $(10 \mathrm{~mL})$ and stirred until the solid was completely dissolved. This solution was added to a $50 \mathrm{~mL}$ reactor with a polytetrafluoroethylene liner. The mixture was hydrothermally reacted for 24 hours in a blast drying oven at $483 \mathrm{~K}$, and the resulting MIL-96(Al) product was washed three times with pure water and dried in an oven at $333 \mathrm{~K}[35]$.

2.3. Characterization. The microstructure of the sample was observed using an S-4800 field-emission scanning electron microscope (FE-SEM). Transmission electron microscopy (TEM) imaging analysis was performed using a Tecnai 12 instrument. X-ray diffraction (XRD) was performed using a Bruker D8 Advance diffractometer to investigate the crystal structure of the sample. The surface properties and valence states of the samples were determined using an ESCALAB 250Xi X-ray photoelectron spectrometer. The Autosorb-i Q3 specific surface area and pore size analyzer and the ZRY-2P thermogravimetric analyzer were used to determine the specific surface area (BET) and produce thermal analysis (TG) data, respectively. Infrared (IR) testing of samples was performed on a VERTEX 70.

2.4. Fluoride Adsorption Experiment. To explore the adsorption performance of the sample in defluorination, a series of 
experiments were carried out. In these experiments, the fluoride-containing wastewater we used was prepared using sodium fluoride and pure water. The solution was filtered using a $0.45 \mu \mathrm{m}$ membrane syringe filter before measuring the fluoride concentration in the water and determined by spectrophotometry with an Alizarin complexone, and the absorbance value was measured using an ultraviolet-visible spectrophotometer (UV755B, Precision Scientific Instrument, Shanghai) at a wavelength of $620 \mathrm{~nm}$. Finally, the fluoride concentration in the solution was obtained according to the standard curve of fluoride. To investigate the effect of the dosage of MIL-96(Al) in defluoridation, different quality MIL-96(Al) was added to $\mathrm{F}^{-}$solution with a concentration of $10 \mathrm{mg} \mathrm{L}^{-1}$, respectively. The solution was placed in a constant temperature shaker shaking at $298 \mathrm{~K}$ at $150 \mathrm{rpm}$ for 2.5 hours and then filtered to determine the absorbance value to calculate the removal rate.

The initial concentration of fluoride in the water had a different effect on the adsorption of MIL-96(Al). Therefore, $0.5 \mathrm{~g} \mathrm{~L}^{-1}$ adsorbent was added to the $\mathrm{F}^{-}$solution with a concentration of 5 to $50 \mathrm{mg} \mathrm{L}^{-1}$, shaking at $298 \mathrm{~K}$ at $150 \mathrm{rpm}$ for 2.5 hours. The $\mathrm{pH}$ may also have effects on the adsorption of the adsorbent, so $0.5 \mathrm{~g} \mathrm{~L}^{-1}$ of MIL-96(Al) was added to the $\mathrm{F}^{-}$solution with a concentration of $10 \mathrm{mg} \mathrm{L}^{-1}$. The $\mathrm{pH}$ of the solution was adjusted to $3-11$ by $\mathrm{HCl}$ and $\mathrm{NaOH}$. At the same time, another set of experiments was carried out, and the common anions of $\mathrm{Cl}^{-}, \mathrm{SO}_{4}{ }^{2-}, \mathrm{NO}_{3}{ }^{-}$, and $\mathrm{CO}_{3}{ }^{2-}$ were quantitatively added to the $\mathrm{F}^{-}$solution with a concentration of $10 \mathrm{mg} \mathrm{L}^{-1}$ with different concentrations of anions, and $0.5 \mathrm{gL}^{-1}$ of MIL-96 (Al) was added. The three sets of experiments were shaken at $298 \mathrm{~K}$ at $150 \mathrm{rpm}$ for 2.5 hours and then filtered to determine the absorbance value to investigate the effect.

The measurement of the adsorption isotherm of the fluoride was carried out in a $250 \mathrm{~mL}$ Erlenmeyer flask. $0.5 \mathrm{~g} \mathrm{~L}^{-1}$ of MIL-96 (Al) was added to the $\mathrm{F}^{-}$solution with a concentration range of 5 to $150 \mathrm{mg} \mathrm{L}^{-1}$ solutions separately. The solutions were shaken at $150 \mathrm{rpm}$ for 2.5 hours at $298 \mathrm{~K}, 303 \mathrm{~K}$, and $308 \mathrm{~K}$, respectively. The Freundlich and Langmuir isothermal models are two widely used adsorption isotherm models $[36,37]$. The D-R isotherm model is proposed by Dubinin and Radushkevich, which is also often used to study whether the adsorption process is physical or chemical adsorption. Therefore, these three models were used to analyze the adsorption process [38].

$$
\text { Freundlich model : } \begin{aligned}
\lg q_{e} & =\lg K+\frac{1}{n} \lg c_{e}, \\
\text { Langmuir model }: \frac{c_{e}}{q_{e}} & =\frac{1}{q^{0} b}+\frac{c_{e}}{q^{0}}, \\
\text { D - R model : } \ln q_{e} & =\ln q^{0}-\beta \varepsilon^{2}, \\
\varepsilon & =R T \ln \left(1+\frac{1}{c_{e}}\right), \\
E & =\frac{1}{\sqrt{(2 \beta)}},
\end{aligned}
$$

where $c_{e}\left(\mathrm{mg} \mathrm{L}^{-1}\right)$ is the concentration of fluoride remaining in the solution when the adsorption equilibrium is reached, $q_{e}\left(\mathrm{mgg}^{-1}\right)$ is the equilibrium adsorption amount, $q^{0}\left(\mathrm{mg} \mathrm{g}^{-1}\right)$ is the single-layer saturated adsorption amount, and $K, n$, and $b$ are constants.

In the study of the adsorption kinetics of MIL-96 (Al), $0.5 \mathrm{gL}^{-1}$ of the adsorbent was added to the $\mathrm{F}^{-}$solution with a concentration of $20 \mathrm{mg} \mathrm{L}^{-1}$, and the solution was shaken at $298 \mathrm{~K}$ and $308 \mathrm{~K}$ at $150 \mathrm{rpm}$ for 2.5 hours. The kinetic data in this process was fitted using pseudo first-order, pseudo second-order, and Elovich models.

$$
\begin{aligned}
\text { Pseudo first - order equation : } \frac{d q_{t}}{d t} & =k 1\left(q_{e}-q_{t}\right), \\
\text { After calculus : } \ln \left(q_{e}-q_{t}\right) & =\ln q_{e}-k 1 t, \\
\text { Pseudo second - order equation }: \frac{d q_{t}}{d t} & =k 2\left(q_{e}-q_{t}\right)^{2}, \\
\text { After calculus : } \frac{t}{q_{t}} & =\frac{1}{k 2 q_{e}^{2}}+\frac{t}{q_{e}}, \\
\text { Elovich models : } q_{t} & =\alpha+k_{e} \ln t,
\end{aligned}
$$

where $q_{t}\left(\mathrm{mgg}^{-1}\right)$ is the amount of fluoride adsorbed at time $t, q_{e}\left(\mathrm{mgg}^{-1}\right)$ is the amount of adsorption at equilibrium, $k_{1}\left(\mathrm{~min}^{-1}\right)$ and $k_{2}\left(\mathrm{~g} \mathrm{mg}^{-1} \mathrm{~min}^{-1}\right)$ are the rate constants, and $k_{2} q_{e}^{2}$ represents the initial adsorption rate. Both $k_{e}$ and $\alpha$ are Elovich constants [37].

The thermal selection parameters of the adsorption process can be calculated by the following formula.

$$
\begin{aligned}
& \Delta G^{0}=\Delta H^{0}-T \Delta S^{0}, \\
& \Delta G^{0}=-R T \operatorname{In} K_{c},
\end{aligned}
$$

where $K_{\mathrm{c}}$ is the equilibrium constant at a certain temperature, $K_{\mathrm{c}}=q_{e} / c_{e}$, indicating the affinity of adsorption. So the above formula can be combined to get

$$
\ln \frac{q_{e}}{c_{e}}=-\frac{-\Delta H^{0}}{R T}+\frac{\Delta S^{0}}{R} .
$$

$\Delta G^{0}, \Delta H^{0}$, and $\Delta S^{0}$ represent the free energy, free enthalpy change, and free entropy change, respectively, and can be plotted by $\ln \left(q_{e} c_{e}{ }^{-1}\right)$ versus $1 / T$, and then, the Gibbs free energy change $\Delta G^{0}$ is obtained according to the obtained slope and intercept [39].

2.5. Regeneration of $M I L-96(A l)$. Regeneration of the adsorbent can greatly reduce costs. Therefore, the heating desorption regeneration method and solvent method were applied for regeneration [40]. The adsorbent used was separated by a centrifuge and then placed in an oven. The heating desorption regeneration method involved heating the spent adsorbent at different temperatures for 12 hours. The solvent method involved soaking the spent adsorbent with $\mathrm{NaOH}$ solution, $\mathrm{HCl}$ solution, $\mathrm{H}_{2} \mathrm{SO}_{4}$ solution, and $\mathrm{HNO}_{3}$ solution 


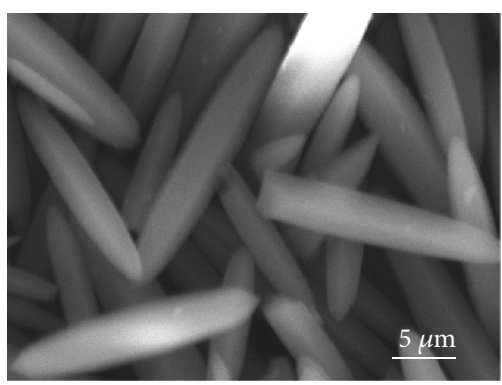

(a)

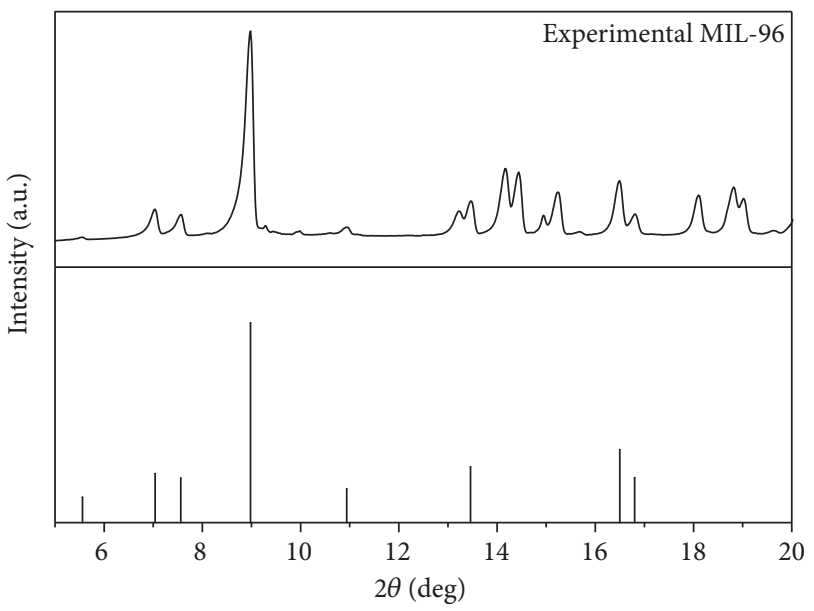

(c)

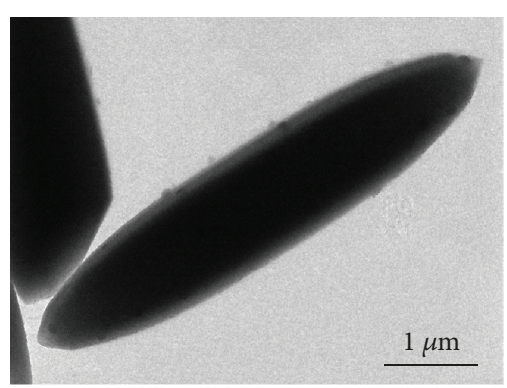

(b)

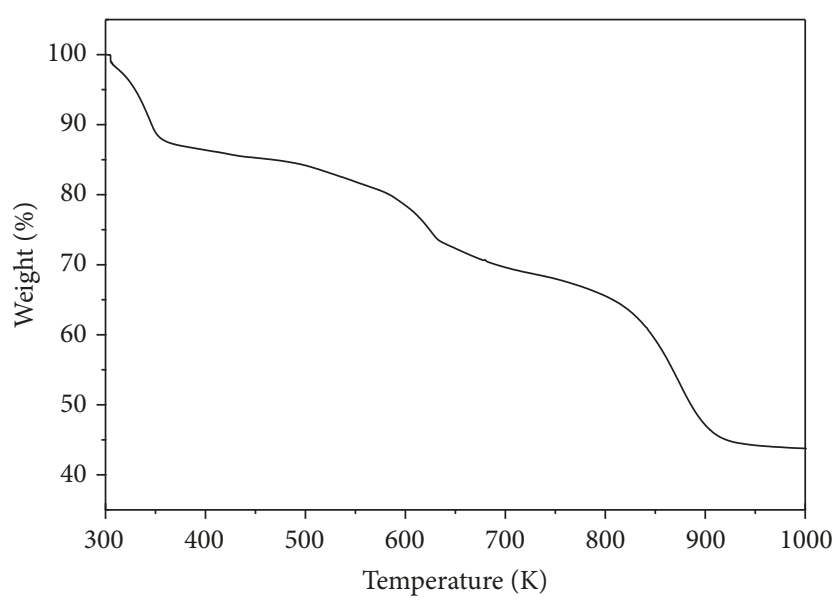

(d)

Figure 1: The SEM image of MIL-96(Al) (a), the TEM image of MIL-96(Al) (b), the XRD patterns of MIL-96(Al) (c), and the loss of TG for MIL-96(Al) (d).

for 12 hours. The adsorbent was then collected and washed by centrifugation.

\section{Results and Discussion}

3.1. Characterization of a Rice Granular Defluorination Adsorbent. To obtain a high-performance defluorination adsorbent, the prepared material was analyzed to verify that the material was MIL-96(Al). The material should have similar characteristics to rice: smooth, uniform in size, and highly crystalline [35]. The SEM image of the prepared MIL-96(Al) (Figure 1(a)) shows that the prepared material had good dispersibility, a similar morphology to rice grains with a uniform size, a regular shape and smooth surface, and a length of $4-6 \mu \mathrm{m}$ and width of $1-2 \mu \mathrm{m}$. Figure $1(\mathrm{~b})$ is the TEM image of the material, which shows that the sample was a solid structure, similar to the SEM image, and was used to preliminarily determine that the prepared material was MIL-96(Al).

Figure 1(c) shows the XRD pattern of the material; the peak intensity was high, indicating high crystallinity; this is supported by the SEM image of the material. The material was indexed to the structure of the MIL-96 $\left(\mathrm{Al}_{12} \mathrm{O}(\mathrm{OH})_{18}\left(\mathrm{H}_{2} \mathrm{O}\right)_{3}\left(\mathrm{Al}_{2}(\mathrm{OH})_{4}\right)(\mathrm{BTC})_{6} \cdot 24 \mathrm{H}_{2} \mathrm{O}\right)$ [35]. In previous studies, MIL-96(Al) was successfully prepared by the solvothermal method and the peak value of the XRD pattern corresponds well to the value in this study, indicating that the crystal form was well prepared. Figure 1(d) presents the TG curve of MIL-96(Al) under $\mathrm{N}_{2}$. As the temperature was initially increased and the boiling point of 1,3,5-benzenetricarboxylic acid is about $583 \mathrm{~K}$, the mass loss $(\sim 15 \%)$ below $373 \mathrm{~K}$ was mainly attributed to the evaporation of free water and the loss $(\sim 5 \%)$ between 373 and $583 \mathrm{~K}$ was the precipitation of bound water. As the temperature reached the boiling point of 1,3,5-benzenetricarboxylic acid, the organic skeleton of the material began to decompose; during this process, mass loss was not obvious. However, when the temperature reached $823 \mathrm{~K}$, the structure collapsed, as seen in the image. This indicated that the material was relatively stable at the typical temperatures used to treat wastewater. The BET surface area was about $220 \mathrm{~m}^{2} \mathrm{~g}^{-1}[33,35]$.

X-ray photoelectron spectroscopy (XPS) was employed to investigate the valence state of different elements in MIL96(Al). It determined that the prepared material contained the four elements Al, N, C, and O (Figure 2(a)). Figure 2(b) shows that $\mathrm{Al} 2 \mathrm{p}$ peaks appeared at $74.75-74.85 \mathrm{eV}$, indicating that the $\mathrm{Al}$ present was trivalent and was $\mathrm{Al}$ oxide, matching the binding energy of $\mathrm{Al}^{+3}$ well [41]. The peak of $\mathrm{O} 1 \mathrm{~s}$ in the metal oxide is between 528 and $531 \mathrm{eV}$, which is the $\mathrm{O}$ in $\mathrm{Al}$ oxide (Figure 2(c)) [34]. It can also clearly see both the aliphatic carbon (at ca. $285 \mathrm{eV}$ ) and the two kinds of strongly oxidized carbon at the higher binding energies up to ca. $289 \mathrm{eV}$ (Figure 2(d)). There are three different kinds of 


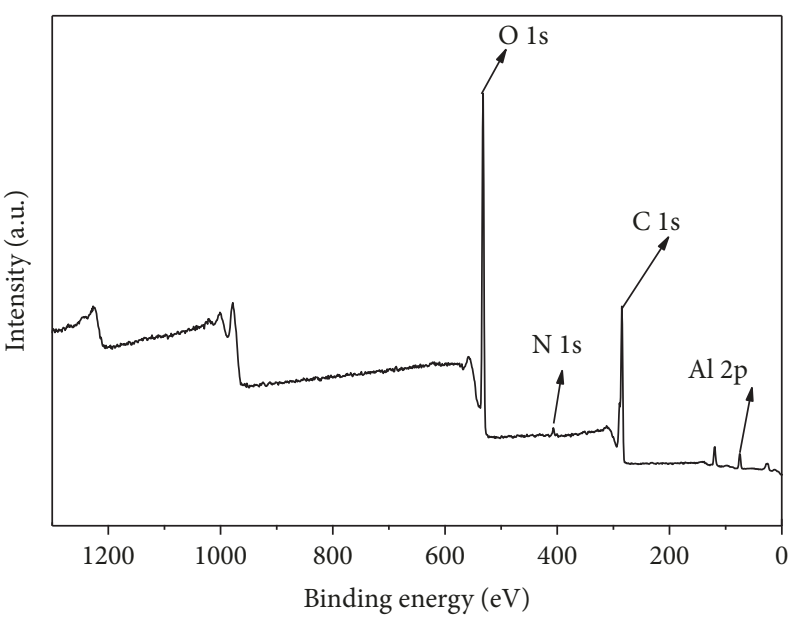

(a)

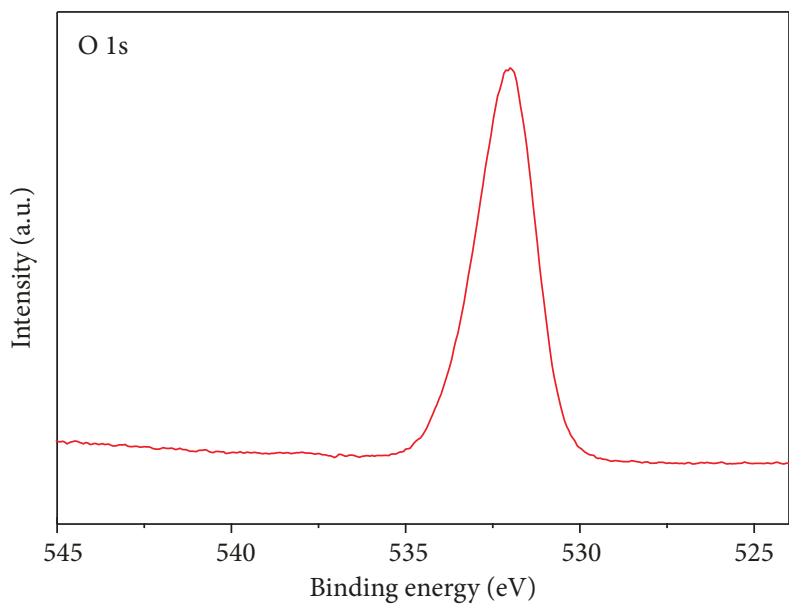

(c)

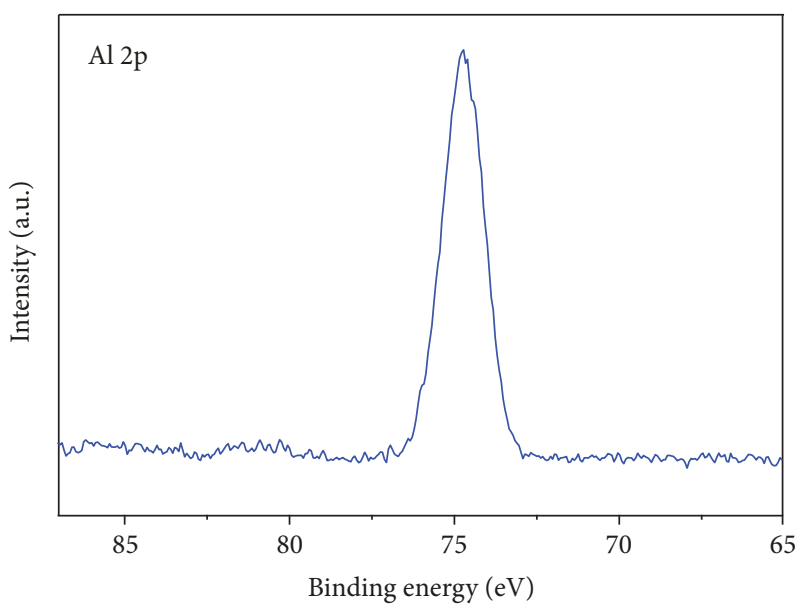

(b)

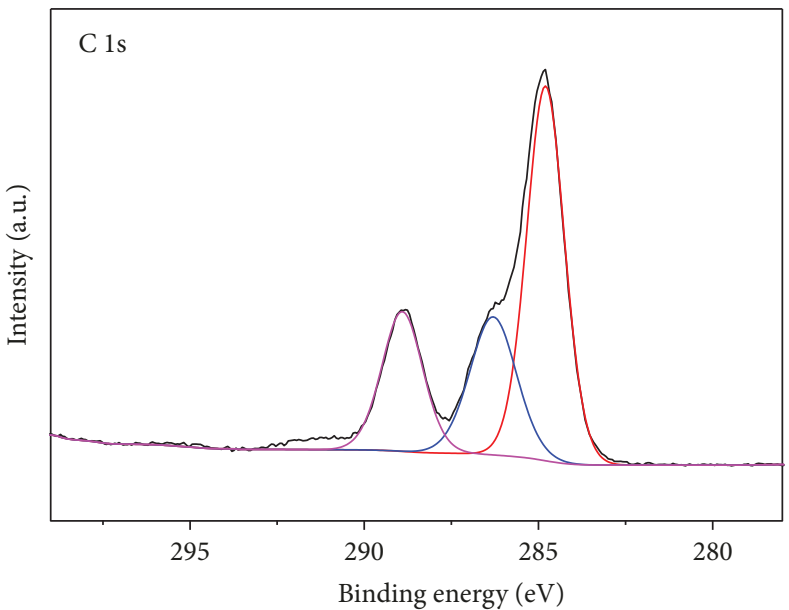

(d)

FIgure 2: The XPS spectra of MIL-96(Al) (a), Al 2p (b), O 1s (c), and C 1s (d) for MIL-96(Al).

carbon in MIL-96(Al): (a) the carbon in the phenyl ring, (b) COO carboxylate groups connected to aluminum cations, and (c) likely carbon of $\mathrm{COOH}$ groups. The last probably constitutes an impurity in MIL-96(Al) due to the BTC precursor.

3.2. Experimental Study on MIL-96(Al) Fluoride Adsorption. The equilibration time for maximum adsorption of fluoride and the effect of dosage and initial concentration on the defluoridation were determined. The removal efficiency was relatively high during the first 50 minutes, and the removal ended after approximately 90 minutes (Figure 3(a)). It is possible that there were more adsorption sites and fluorion on the surface when the adsorbent was added, and the adsorption rate rose rapidly. When the dosage of the adsorbent remains unchanged, the higher concentration will promote the competitive combination of fluorion and the adsorption site, and the adsorption saturation will be reached more quickly. Furthermore, this phenomenon indicated that the surface of MIL-96( $\mathrm{Al})$ is heterogeneous and rich in activated binding sites [42]. And in Figure 3(b), it can be seen that the dosage of the adsorbent $0.5 \mathrm{gL}^{-1}$ was suitable for the treatment of $10 \mathrm{~m} \mathrm{~L} \mathrm{~L}^{-1}$ of fluoride solution.

Figure 4(a) shows the system under different $\mathrm{pH}$ conditions. Under acidic conditions, the removal rate reaches $100 \%$ because in the acidic environment, some $\mathrm{F}^{-}$will react with $\mathrm{H}^{+}$in water. The formation of $\mathrm{HF}$, in the form of molecules in water, coupled with the action of the adsorbent, can achieve a $100 \%$ removal rate. Interestingly, under alkaline conditions, the removal rate also increased. It is possible that the hydroxide in the solution combines with the $\mathrm{Al}$ ions, bridging the organic skeleton of the material causing flocculation, which then causes the fluorion in the water to separate from the liquid phase, resulting in a significant increase in the removal rate [43]. Similarly, blank experiments adjusting the $\mathrm{pH}$ were carried out. Under acidic conditions, the concentration of fluoride in the solution was significantly reduced, while the concentration did not change significantly under alkaline conditions. However, during the treatment of wastewater with a strong acid and alkali, the acid and alkali were generally recovered first; the $\mathrm{pH}$ is controlled and then treated, so the adsorbent is not affected. The effect of 


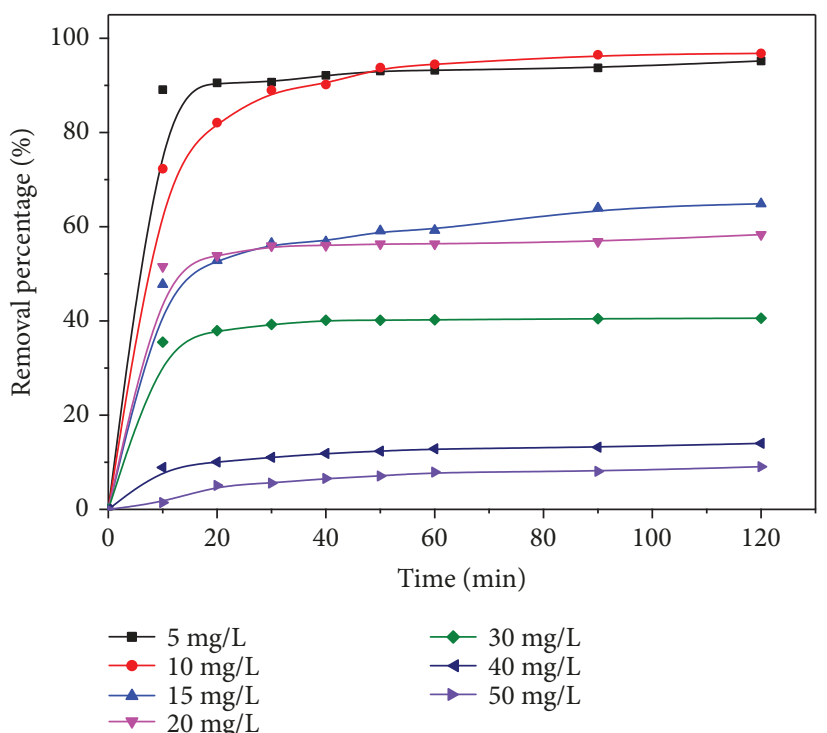

(a)

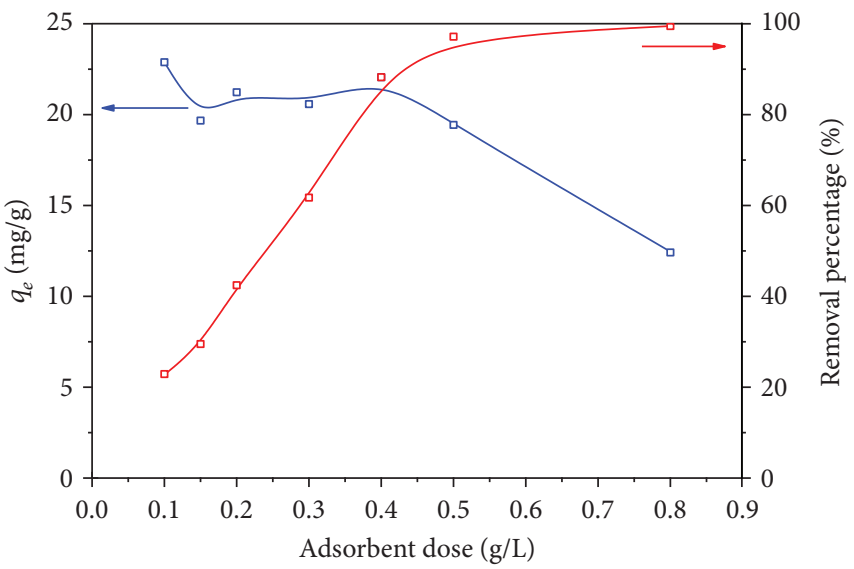

(b)

Figure 3: The effect of concentration on removal percentage when the dosage of MIL-96(Al) is $0.5 \mathrm{~g} / \mathrm{L}$ at about $298 \mathrm{~K}$ (a) and the effect of adsorbent dosage on the adsorption capacity and removal percentage of MIL-96(Al) to fluoride when the concentration is $10 \mathrm{mg} / \mathrm{L}$ (b).

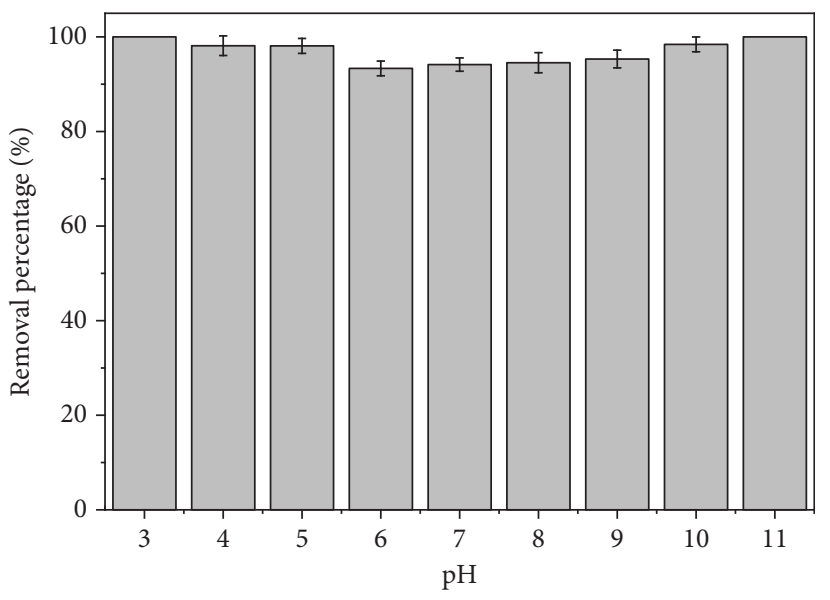

(a)

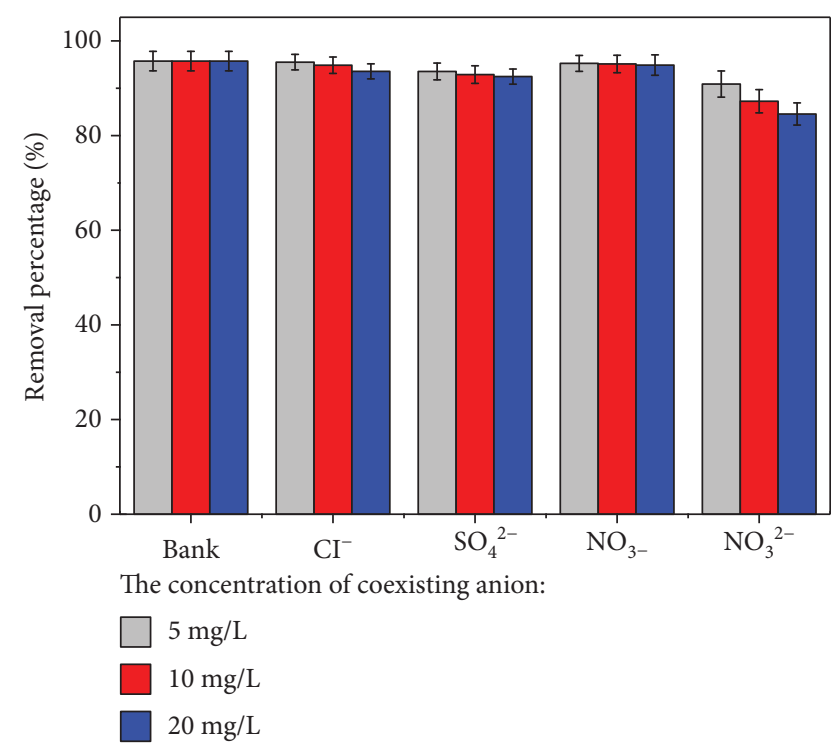

(b)

FIGURE 4: The effect of $\mathrm{pH}$ on removal percentage (a) and the effect of anions on the removal rate (b) (the dosage of MIL-96(Al): $0.5 \mathrm{~g} \mathrm{~L}{ }^{-1}$, the concentration of fluoride: $10 \mathrm{mg} \mathrm{L}^{-1}$ ).

coexisting anions on the removal rate can be seen in Figure 4(b). $\mathrm{CO}_{3}{ }^{2-}$ interfered with fluoride adsorption through the competition of fluoride active adsorption sites on MIL-96(Al), and high concentration of $\mathrm{CO}_{3}{ }^{2-}$ led to a decrease in fluoride adsorption efficiency, but the actual impact was not very big. Other common coexisting anions only had very small effect on the fluoride removal of the adsorbent, indicating that MIL-96(Al) had higher selectivity for removing fluoride and stronger anti-interference ability.
3.3. Isothermal Adsorption Model of MIL-96(Al). The variation in the equilibrium adsorption amount with the equilibrium concentration is described by the adsorption isotherm. The adsorption isotherms shed light on the interaction between the adsorbate and adsorbent and were therefore determined for this system (Table 1 and Figure 5) and could be judged from the line type as the Langmuir isotherm. As the initial concentration increases, so too does the amount of adsorption; also, an increase in temperature increases the adsorption capacity of the adsorbent. 
TABLE 1: The data of isotherm at different temperatures.

\begin{tabular}{|c|c|c|c|c|c|c|}
\hline \multirow{2}{*}{$C_{0}\left(\mathrm{mg} \mathrm{L}^{-1}\right)$} & \multicolumn{2}{|c|}{$298 \mathrm{~K}$} & \multicolumn{2}{|c|}{$303 \mathrm{~K}$} & \multicolumn{2}{|c|}{$308 \mathrm{~K}$} \\
\hline & $C_{e}\left(\mathrm{mg} \mathrm{L}^{-1}\right)$ & $q_{e}\left(\mathrm{mgg} \mathrm{g}^{-1}\right)$ & $C_{e}\left(\mathrm{mg} \mathrm{L}^{-1}\right)$ & $q_{e}\left(\mathrm{mg} \mathrm{g}^{-1}\right)$ & $C_{e}\left(\mathrm{mg} \mathrm{L}^{-1}\right)$ & $q_{e}\left(\mathrm{mg} \mathrm{g}^{-1}\right)$ \\
\hline 5.00 & 0.00 & 10.00 & 0.00 & 10.00 & 0.00 & 10.00 \\
\hline 10.00 & 0.33 & 19.35 & 0.32 & 19.36 & 0.00 & 20.00 \\
\hline 15.00 & 4.77 & 20.47 & 3.34 & 23.32 & 1.62 & 26.76 \\
\hline 20.00 & 8.38 & 23.24 & 7.84 & 24.32 & 4.97 & 30.06 \\
\hline 30.00 & 16.82 & 26.36 & 16.25 & 27.50 & 14.59 & 30.82 \\
\hline 40.00 & 25.90 & 28.20 & 25.25 & 29.50 & 22.92 & 34.15 \\
\hline 50.00 & 35.45 & 29.10 & 33.38 & 33.23 & 31.37 & 37.27 \\
\hline 60.00 & 43.79 & 32.42 & 43.06 & 33.87 & 40.88 & 38.24 \\
\hline 80.00 & 62.29 & 35.42 & 61.57 & 36.86 & 59.57 & 40.86 \\
\hline 100.00 & 81.35 & 37.30 & 79.86 & 40.28 & 79.09 & 41.83 \\
\hline 120.00 & 99.85 & 40.30 & 98.75 & 43.15 & 98.66 & 44.68 \\
\hline 150.00 & 129.75 & 40.50 & 128.36 & 43.28 & 127.58 & 44.83 \\
\hline
\end{tabular}

$C_{0}\left(\mathrm{mg} \mathrm{L}^{-1}\right)$ is the initial concentration of fluoride in the solution, $C_{e}\left(\mathrm{mg} \mathrm{L}^{-1}\right)$ is the concentration of fluoride remaining in the solution, and $q_{e}\left(\mathrm{mg} \mathrm{g}^{-1}\right)$ is the equilibrium adsorption amount.

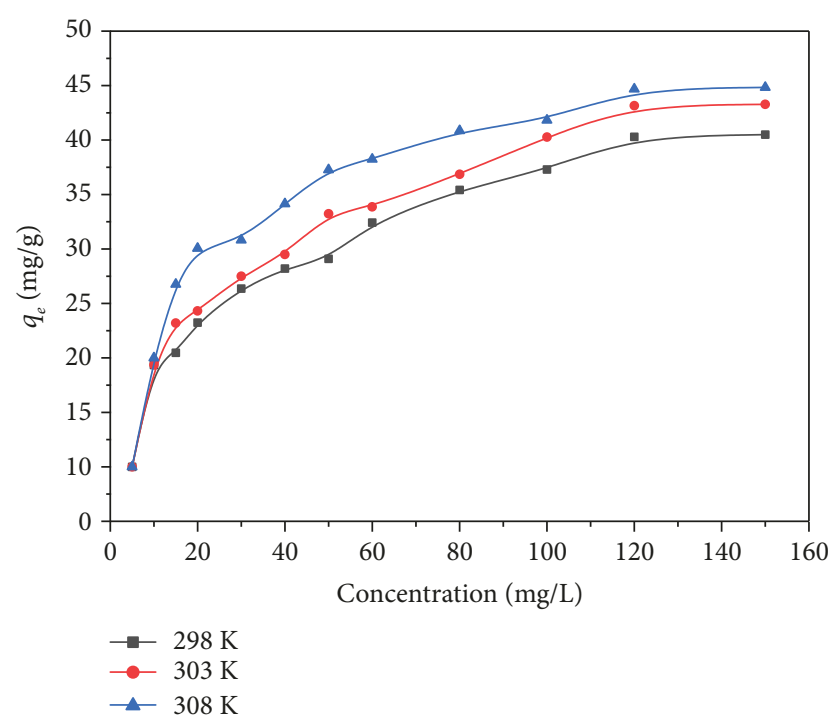

FIGURE 5: The adsorption isotherms of $\mathrm{F}$ under different reaction temperatures.

The adsorption process was analyzed by three adsorption isotherm models, Freundlich, Langmuir, and D-R models, in detail. Tables 2 and 3 present the fitting results of the Freundlich and Langmuir models. The correlation coefficient $R^{2}$ of the Langmuir model was greater than that of the Freundlich model, demonstrating that the adsorption of fluoride by MIL-96(Al) was more consistent with the Langmuir model and suggesting monolayer adsorption. At the same time, according to the shape of the adsorption isotherm, it can be judged as the Langmuir model. Although the Freundlich model is poorly correlated, the value of $1 / n$ is also between 0.1 and 0.5 , indicating that the adsorption process is also prone to occur. Furthermore, the value of $1 / n$ decreased with increasing temperature, which indicated that higher temperature assisted the adsorption process. The theoretical maxi- mum value for fluoride adsorption was $42.194 \mathrm{mgg}^{-1}$ at $298 \mathrm{~K}$. In the Langmuir model, $q^{0}$ and $b$ are characteristic parameters of adsorption performance. The larger $q^{0}$ is, the larger the adsorption capacity is under the same conditions, and the larger $b$ is, the faster the adsorption rate is. With the increase in temperature, $q^{0}$ and $b$ were also increased. The adsorption capacity and adsorption rate of MIL-96(Al) increased. $R_{\mathrm{L}}$ also is an important dimensionless constant separation factor in the Langmuir model, and it indicates the shape of the isotherm accordingly and can be expressed by the following formula: $R_{\mathrm{L}}=1 /\left(1+b \times c_{0}\right)$, where $b$ is a constant and $c_{0}$ is the initial concentration of fluoride. When $0<R_{\mathrm{L}}<1$, adsorption readily proceeds, whereas when $R_{\mathrm{L}}=0$, adsorption is an irreversible process. When $R_{\mathrm{L}}=1$, the isotherm is linear and adsorption is reversible, and when $R_{\mathrm{L}}>1$, the adsorption process is difficult to verify [44]. The $R_{\mathrm{L}}$ values under different concentration conditions were between 0 and 1 , indicating that the adsorption process was relatively easy. The D-R isothermal model does not assume a uniform adsorbent surface or a constant adsorption energy. Based on the fitting results of the Freundlich and Langmuir models, it can be determined whether there was physical or chemical adsorption. When $E=8-16 \mathrm{~kJ} \mathrm{~mol}^{-1}$, the adsorption process is triggered by ion exchange; when $E<8 \mathrm{~kJ} \mathrm{~mol}^{-1}$, the adsorption process is physical adsorption and physical forces such as van der Waals and hydrogen bonding may affect the adsorption mechanism; and when $E>16 \mathrm{~kJ} \mathrm{~mol}^{-1}$, the adsorption process is chemical adsorption. Table 4 presents the fitting result of the D-R isothermal model. The average adsorption energy was during $8-16 \mathrm{~kJ} \mathrm{~mol}^{-1}$, showing that the adsorption is mainly caused by ion exchange during the process [38].

3.4. Adsorption Kinetics of MIL-96(Al). Another important indicator of the adsorption capacity of adsorbents on adsorbates is the adsorption rate. Figure 6(a) shows that, initially, the adsorption of fluoride onto MIL-96(Al) increases rapidly, 
TABLE 2: Fitted result by the Freundlich model at different temperatures.

\begin{tabular}{|c|c|c|c|c|}
\hline \multirow[b]{2}{*}{ Temperature $(\mathrm{K})$} & \multicolumn{3}{|c|}{ Parameter } & \multirow{2}{*}{$\begin{array}{c}\text { Linear equation } \\
\lg q_{e}=\lg K+\left(\frac{1}{n}\right) \lg c_{e}\end{array}$} \\
\hline & K & $\frac{1}{n}$ & $R^{2}$ & \\
\hline 298 & 14.4644 & 0.1777 & 0.9849 & $\lg q_{e}=0.2134 \lg c_{e}+1.1603$ \\
\hline 303 & 16.9785 & 0.2564 & 0.9581 & $\lg q_{e}=0.1906 \lg c_{e}+1.2299$ \\
\hline 308 & 24.1434 & 0.1250 & 0.9486 & $\lg q_{e}=0.1250 \lg c_{e}+1.3827$ \\
\hline
\end{tabular}

TABLE 3: Fitted results by the Langmuir model at different temperatures.

\begin{tabular}{lcccc}
\hline Temperature $(\mathrm{K})$ & Parameter & Linear equation & & \\
\hline 298 & $b$ & $q^{0}\left(\mathrm{mg} \mathrm{Fg}^{-1}\right)$ & $R^{2}$ & $\frac{c_{e}}{q_{e}}=\left(\frac{1}{q^{0} b}\right)+\left(\frac{c_{e}}{q^{0}}\right)$ \\
303 & 0.1139 & 42.1941 & 0.9880 & $\frac{c_{e}}{q_{e}}=0.0237 c_{e}+0.2080$ \\
308 & 0.1220 & 44.8430 & 0.9877 & $\frac{c_{e}}{q_{e}}=0.0223 c_{e}+0.1828$ \\
\hline
\end{tabular}

TABLE 4: Fitted result by the D-R model at different temperatures.

\begin{tabular}{|c|c|c|c|c|}
\hline Temperature (K) & $E\left(\mathrm{~kJ} \mathrm{~mol}^{-1}\right)$ & $\begin{array}{l}\text { Parameter } \\
q^{0}\left(\mathrm{mg} \mathrm{Fg}^{-1}\right)\end{array}$ & $R^{2}$ & $\begin{array}{c}\text { Linear equation } \\
\ln q_{e}=\ln q^{0}-\beta \varepsilon^{2}\end{array}$ \\
\hline 298 & 11.6248 & 41.4381 & 0.8704 & $\ln q_{e}=-0.0037 \varepsilon^{2}+3.7242$ \\
\hline 303 & 11.7851 & 44.4916 & 0.9039 & $\ln q_{e}=-0.0036 \varepsilon^{2}+3.7953$ \\
\hline 308 & 11.7851 & 46.0211 & 0.9638 & $\ln q_{e}=-0.0036 \varepsilon^{2}+3.8291$ \\
\hline
\end{tabular}

with the adsorption amount reaching $80 \%$ or more of the equilibrium adsorption amount after 20 minutes. As the oscillation time continued to increase, the adsorption amount increased slowly. When the oscillation time reached $90 \mathrm{~min}$, no significant change in the adsorption amount was observed; therefore, under the experimental conditions, the adsorption equilibrium time of fluoride adsorption onto MIL-96(Al) was determined to be $90 \mathrm{~min}$ [45]. It can be seen from the figure that as the temperature increases, both the adsorption rate and the adsorption capacity increase, indicating that the process is an endothermic process.

The dynamic process was fitted using the pseudo firstorder equation, pseudo second-order equation, and Elovich model (Figure 6(b)-6(d)). The parameters obtained by fitting the above three kinetic models are shown in Table 5. The fitting results obtained by the three models show a good correlation; however, the relative error of the $R^{2}$ of the pseudo first-order kinetics fit was bigger. Therefore, this adsorption process did not follow the pseudo first-order equation. The linear correlation coefficient of the pseudo second-order equation fitting is close to 1 (0.9989), and the relative error of the equilibrium adsorption amount is small, which is better than the fitting result of the pseudo first-order equation. Therefore, the adsorption process was determined to follow the pseudo second-order equation and it is mainly chemical adsorption.

3.5. Adsorption Thermodynamics of MIL-96(Al). Adsorption thermodynamics, which investigates the adsorption capacity with a change in the adsorption temperature, is important for determining the adsorption capacity; thus, it is used in practical applications. From Table $6, \Delta H^{0}$ was shown to be a positive number. It is known that the adsorption process for fluoride is endothermic, and it was experimentally verified that an increase in temperature was advantageous for adsorption to the adsorbent surface and the process is endothermic in kinetic studies. $\Delta S^{0}$ was also shown to be a positive number, meaning that the irregular interlayer adsorption of the solid-liquid system interface is enhanced during the adsorption of fluoride and the disorder of the adsorbateadsorbent system is increased. $\Delta S^{0}>0$ indicates that the adsorption reaction is an entropy-driven process. For solidliquid exchange adsorption, the exchange of solute molecules from the liquid phase to the solid-liquid interface loses some of the free energy, resulting in a decrease in entropy. Therefore, the adsorption of fluoride ions onto the surface of the adsorbent should also be a process of entropy reduction, but the result of this experiment is that the entropy is less 


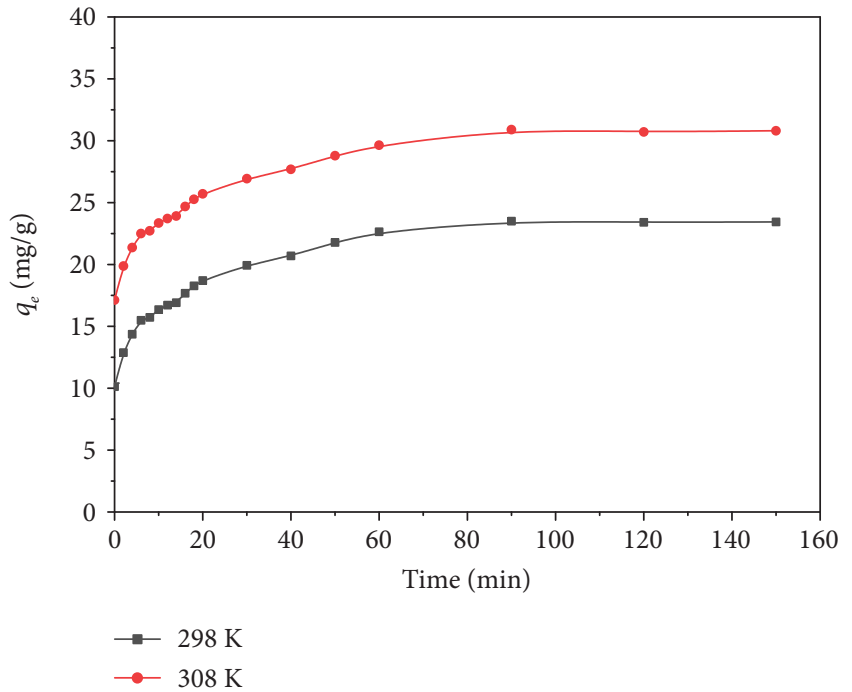

(a)

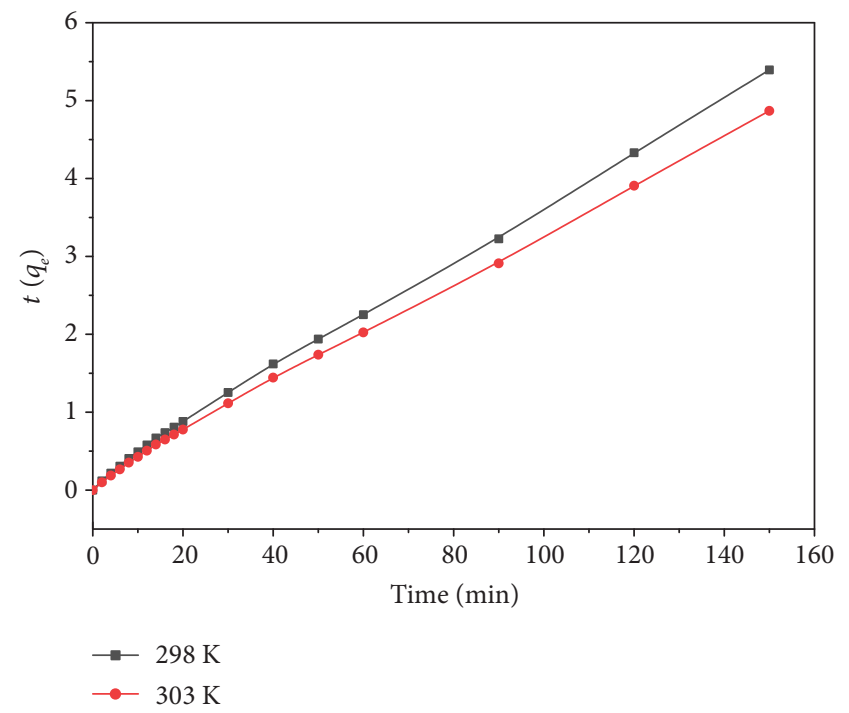

(c)

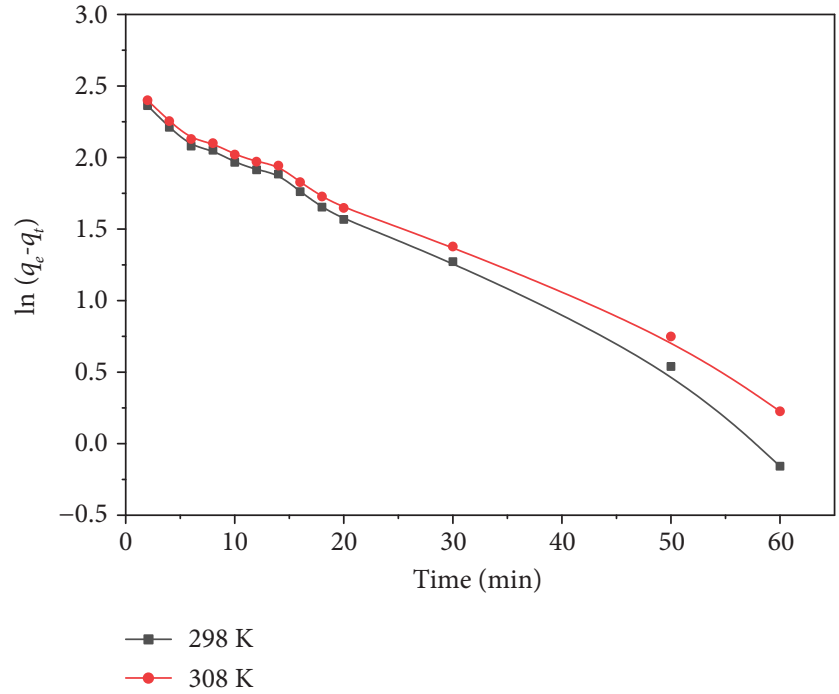

(b)

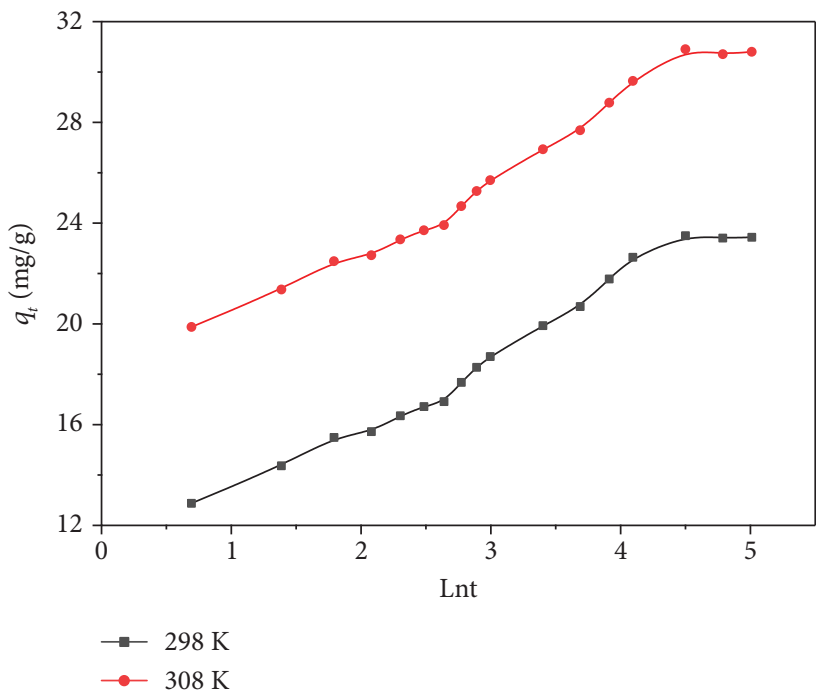

(d)

Figure 6: (a) Adsorption rate curves of fluoride. (b) Fitted result by the pseudo first-order equation. (c) Fitted result by the pseudo secondorder equation in the second-order kinetic model. (d) Fitted result by the Elovich model.

than 0 , so that in the adsorption process, other substances must be released to increase the entropy value. The release of matter causes the increase in entropy to cancel out the decrease in entropy caused by fluoride ion adsorption, causing the final smoke of the system to become positive. It is also possible to introduce that the adsorption process is an ion exchange process. $\Delta G^{0}$ was shown to be smaller than zero, meaning that the reaction process was spontaneous; however, it is not in the standard state for practical applications and physical adsorption also existed in the case of chemical adsorption. Increasing the temperature lowers $\Delta G^{0}$, indicating that the spontaneity of the adsorption process was proportional to the temperature [44].

3.6. Regeneration of MIL-96(Al). The experimental study found that traditional regeneration, such as of hydrochloric acid, sodium hydroxide, and ethanol, did not achieve the desired effect on material regeneration and a higher concentration of $\mathrm{NaOH}$ had a better regeneration effect. In this study, $0.1 \mathrm{~mol} \mathrm{~L}^{-1}$ and $0.2 \mathrm{~mol} \mathrm{~L}^{-1} \mathrm{NaOH}$ solution were used as the analytical solution to soak the saturated adsorbent for $12 \mathrm{~h}$ and yielded promising results. The removal performance of MIL-96(Al) in seven cycles was shown in Figure 7. It can be seen that as the number of regenerations increased, the volume followed a downward trend. After being used seven times, the fluoride removal rate dropped to $\sim 61.8 \%$ and the removal effect decreased by $\sim 40 \%$, as $\mathrm{OH}^{-}$in the solution exchanged with $\mathrm{F}^{-}$on the adsorbent, transferring the fluorion into the analytical solution. An increase in the concentration of the solution could also contribute to the rate of exchange. However, the analytical solution is strongly alkaline and the $\mathrm{Al}$ on the adsorbent will 
TABLe 5: Fitted results by different kinetic models.

\begin{tabular}{|c|c|c|c|c|c|}
\hline \multirow[t]{2}{*}{ Dynamic mode } & \multicolumn{4}{|c|}{ Parameter } & \multirow[b]{2}{*}{ Kinetic equation } \\
\hline & $K$ & $k_{1}$ & $q_{e}\left(\mathrm{mgg}^{-1}\right)$ & $R^{2}$ & \\
\hline \multirow[t]{3}{*}{ Pseudo first-order equation } & 298 & 0.0400 & 10.9539 & 0.9895 & $\ln \left(q_{e}-q_{t}\right)=-0.0400 t+2.3937$ \\
\hline & 308 & 0.0349 & 10.9135 & 0.9931 & $\ln \left(q_{e}-q_{t}\right)=-0.0349 \mathrm{t}+2.3900$ \\
\hline & $K$ & $k_{2}$ & $q_{e}\left(\mathrm{mgg}^{-1}\right)$ & $R^{2}$ & Kinetic equation \\
\hline \multirow[t]{3}{*}{ Pseudo second-order equation } & 298 & 0.00942 & 28.4091 & 0.9986 & $\frac{t}{q t}=0.0352 t+0.1315$ \\
\hline & 308 & 0.00971 & 31.4465 & 0.9989 & $\frac{t}{q t}=0.0318 t+0.1041$ \\
\hline & K & $k_{\mathrm{e}}$ & $\alpha$ & $R^{2}$ & Kinetic equation \\
\hline \multirow[t]{2}{*}{ Elovich model } & 298 & 2.7764 & 10.3370 & 0.9776 & $q_{t}=2.7764 \ln t+10.3370$ \\
\hline & 308 & 2.8554 & 17.1600 & 0.9789 & $q_{t}=2.8554 \ln t+17.1600$ \\
\hline
\end{tabular}

TABLE 6: The thermodynamic parameters for the adsorption.

\begin{tabular}{|c|c|c|c|c|c|}
\hline \multirow[b]{2}{*}{$C_{0}\left(\mathrm{mgg}^{-1}\right)$} & \multicolumn{3}{|c|}{ Parameter } & & \\
\hline & $\Delta H^{0}\left(\mathrm{~kJ} \mathrm{~mol}^{-1}\right)$ & $\Delta S^{0}\left(\mathrm{~J} \mathrm{~mol}^{-1} \mathrm{~K}^{-1}\right)$ & \multicolumn{3}{|c|}{$\Delta G^{0}\left(\mathrm{~kJ} \mathrm{~mol}^{-1}\right)$} \\
\hline 20 & 59.263 & 206.578 & -2.297 & -3.330 & -4.363 \\
\hline 30 & 22.718 & 79.770 & -1.053 & -1.451 & -1.850 \\
\hline 40 & 23.861 & 80.537 & -0.139 & -0.542 & -0.944 \\
\hline
\end{tabular}

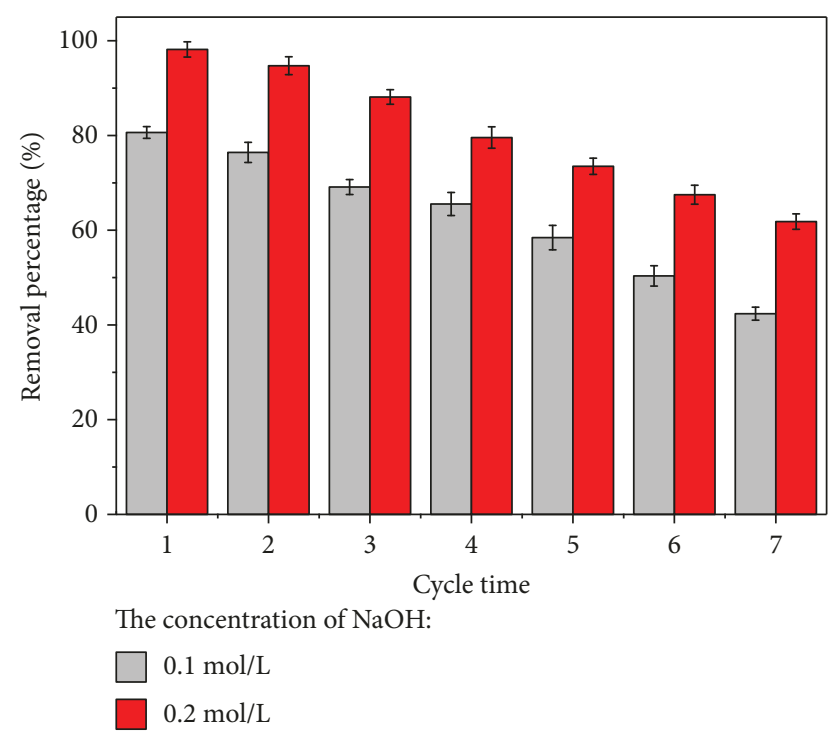

FIGURE 7: Seven consecutive adsorption cycles of MIL-96(Al) (the dosage of MIL-96(Al): $0.5 \mathrm{~g} \mathrm{~L}^{-1}$, the concentration of fluoride and coexisting anion: $\left.10 \mathrm{mg} \mathrm{L}^{-1}\right)$.

undergo a redox reaction with $\mathrm{NaOH}$; therefore, after regeneration, the performance of the adsorbent dropped but was still far better than that of the commercially available activated alumina [45]. The material has a larger specific surface area, higher adsorption capacity, and a convenient synthesis method compared to many other adsorbents (Table 7). At present, there are some adsorbents with outstanding perfor- mance. The preparation of AlFu MOF is more complicated [31], but its excellent performance will become the target of our future research. MIL-96(Al) also has good adsorption performance after regeneration and has shown good potential as a novel fluoride removal adsorbent.

3.7. Adsorption Mechanism. The IR spectrum of MIL-96(Al) before and after adsorption of fluoride is shown in Figure 8(a). This provides insight into the adsorption mechanism of fluoride onto this material. The vibrational band at $1,400-1,600 \mathrm{~cm}^{-1}$ was assigned to the carboxyl functional group before the adsorption of fluoride. For the bound C-O group, $v_{\text {asym }}$ and $v_{\text {sym }}$ were between 1,459 and $1,399 \mathrm{~cm}^{-1}$ and the strong absorption bands were at 1,596 and $1,671 \mathrm{~cm}^{-1}$, indicating that the BTC species was a coordinated $\mathrm{Al}$ atom [35]. There was fluctuation between 1,200 and $1,350 \mathrm{~cm}^{-1}$, and the corresponding peak in the stretching model of $\mathrm{Al}-\mathrm{OH}$ appears at $1,330 \mathrm{~cm}^{-1}$. However, this peak disappeared after adsorption and another distinct characteristic peak appeared at $1,123 \mathrm{~cm}^{-1}$; this was attributed to the formation of Al-F bonds, showing that the absorption of fluoride by this material was mainly dependent on the exchange of - $\mathrm{OH}$ bonded to $\mathrm{Al}$ in the structure of the material [51]. Figure 8(b) shows the possible adsorption process of MIL-96(Al) for fluoride removal. The $\mathrm{F}^{-}$in water substituted $-\mathrm{OH}$ on the adsorbent and combined with the $\mathrm{Al}$ atom to achieve the purpose of defluorination, which was a method by means of metal sites in MOFs to remove fluoride. Similarly, after adsorption, the adsorbent can be regenerated by replacing the $\mathrm{F}^{-}$using -OH supplied by high-concentration $\mathrm{NaOH}$ solution. 
TABLE 7: Comparison of different adsorbents in literatures.

\begin{tabular}{lcccccc}
\hline Adsorbents & MgOC & Activated alumina & MCLRB & MOCA & COCA & MIL-96(Al) \\
\hline Adsorption capacity $\left(\mathrm{mg} \mathrm{Fg}^{-1}\right)$ & 4.44 & 2.07 & 22.35 & 2.85 & 7.77 & 42.19 \\
Synthetic method & Calcination & Calcination & Inverse suspension & Calcination & Calcination & Hydrothermal \\
BET $\left(\mathrm{m}^{2} \mathrm{~g}^{-1}\right)$ & 45.5 & 185.6 & 21.24 & 170.4 & 189.3 & 220.0 \\
Cycle times & N.R. & N.R. & 7 & N.R. & N.R. & 7 \\
Refs. & {$[46]$} & {$[47]$} & {$[48]$} & [49] & [50] & This work \\
\hline
\end{tabular}

N.R.: nonreported; MgOC: magnesia/chitosan composite; MCLRB: mixed-rare earth magnetic chitosan beads; MOCA: manganese-oxide-coated alumina; COCA: copper oxide-coated alumina.

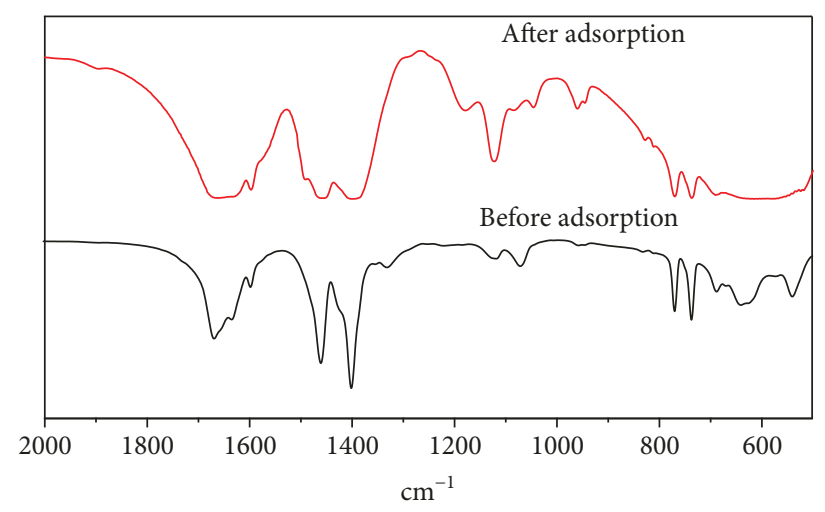

(a)

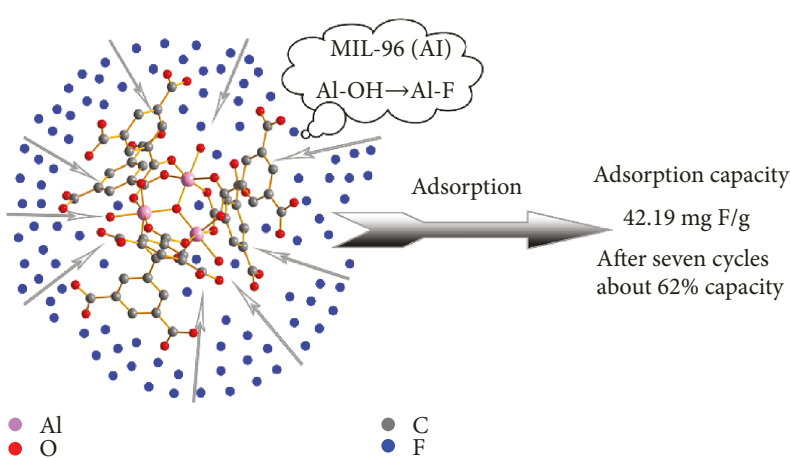

(b)

FIgURE 8: The IR spectra of MIL-96(Al) before and after adsorption of fluoride (a) and the adsorption process of MIL-96(Al) (b).

\section{Conclusions}

The rice-like MIL-96(Al) was fabricated successfully by hydrothermal reaction and showed better fluoride removal performance than many other adsorbents. The adsorption isotherm of MIL-96(Al) matched with the Langmuir model, and the theoretical fluoride adsorption capacity was up to $42.19 \mathrm{mgg}^{-1}$ at $298 \mathrm{~K}$. The process was shown to be monolayer adsorption; the adsorption kinetics and thermodynamics followed pseudo second-order kinetics, typical of an endothermic chemisorption process. The initial concentration affected the defluorination efficiency, but this can be adjusted by controlling the dosage. The $\mathrm{pH}$ of the initial solution had a small effect on the removal of fluoride by MIL-96(Al) and was stable in the $\mathrm{pH}$ range of 6-9. Various common anions in water influenced the removal slightly, indicating that MIL-96(Al) has good selectivity for defluorination. Regeneration of the adsorbent can reduce the application cost significantly. $\mathrm{OH}^{-}$ within the $\mathrm{NaOH}$ solution can exchange the $\mathrm{F}^{-}$on the adsorbent; thus, a $0.2 \mathrm{~mol} \mathrm{~L}^{-1} \mathrm{NaOH}$ solution was used to regenerate the MIL-96(Al) well, which showed good adsorption properties even after seven cycles. Testing indicated that MIL-96(Al) has great potential for defluorination of drinking water and industrial wastewater.

\section{Data Availability}

The data used to support the findings of this study are included within the article.

\section{Conflicts of Interest}

The authors declare that they have no conflicts of interest.

\section{Acknowledgments}

This study was funded by the Social Development Project of Zhenjiang (No. 2016014), the Qing Lan Project for Young Core Teachers in University of Jiangsu Province, the China Postdoctoral Science Foundation (No. 2019M652274), the Foundation from Marine Equipment and Technology Institute for Jiangsu University of Science and Technology, China (HZ20190004), and the Natural Science Foundation of China (Nos. 51908252, 41601304). In addition, the English in this document has been checked by a company that provides professional English language editing services.

\section{References}

[1] J. Cai, X. Zhao, Y. Zhang, Q. Zhang, and B. Pan, "Enhanced fluoride removal by La-doped Li/Al layered double hydroxides," Journal of Colloid and Interface Science, vol. 509, pp. 353-359, 2018.

[2] M. K. So, Y. Miyake, W. Y. Yeung et al., "Perfluorinated compounds in the Pearl River and Yangtze River of China," Chemosphere, vol. 68, no. 11, pp. 2085-2095, 2007.

[3] H. Wu, H. Zhang, Q. Yang, D. Wang, W. Zhang, and X. Yang, "Calcined chitosan-supported layered double hydroxides: an efficient and recyclable adsorbent for the removal of fluoride from an aqueous solution," Materials, vol. 10, no. 11, article 1320, 2017. 
[4] I. N. Dias, J. P. Bassin, M. Dezotti, and V. J. P. Vilar, "Fluorene oxidation by solar-driven photo-Fenton process: toward mild $\mathrm{pH}$ conditions," Environmental Science and Pollution Research, vol. 25, no. 28, pp. 27808-27818, 2018.

[5] A. Bhatnagar, E. Kumar, and M. Sillanpaa, "Fluoride removal from water by adsorption-a review," Chemical Engineering Journal, vol. 171, no. 3, pp. 811-840, 2011.

[6] C. Prathibha, B. Sharma, L. A. A. Chunduri, S. K. Aditha, T. (. M.). Rattan, and K. Venkataramaniah, "Nano calcium aluminum mixed oxide: a novel and effective material for defluoridation of drinking water," Separation Science and Technology, vol. 50, no. 13, pp. 1915-1924, 2015.

[7] H. G. Gorchev and G. Ozolins, "WHO guidelines for drinkingwater quality," WHO Chronicle, vol. 38, no. 3, pp. 104-108, 1984.

[8] Z. Ruan, Y. Tian, J. Ruan et al., "Synthesis of hydroxyapatite/multi-walled carbon nanotubes for the removal of fluoride ions from solution," Applied Surface Science, vol. 412, pp. 578-590, 2017.

[9] M. Mohapatra, S. Anand, B. K. Mishra, D. E. Giles, and P. Singh, "Review of fluoride removal from drinking water," Journal of Environmental Management, vol. 91, no. 1, pp. 67-77, 2009.

[10] S. Ayoob, A. K. Gupta, and V. T. Bhat, “A conceptual overview on sustainable technologies for the defluoridation of drinking water," Critical Reviews in Environmental Science and Technology, vol. 38, no. 6, pp. 401-470, 2008.

[11] M. K. Adak, A. Sen, A. Mukherjee, S. Sen, and D. Dhak, "Removal of fluoride from drinking water using highly efficient nano-adsorbent, $\mathrm{Al}(\mathrm{III})-\mathrm{Fe}(\mathrm{III})-\mathrm{La}$ (III) trimetallic oxide prepared by chemical route," Journal of Alloys and Compounds, vol. 719, pp. 460-469, 2017.

[12] W. X. Gong, J. Qu, R. P. Liu, and H. C. Lan, "Fluoride removal from water by adsorption-a review," Chemical Engineering Journal, vol. 189, pp. 126-133, 2012.

[13] Y. Ku and H. M. Chiou, "The adsorption of fluoride ion from aqueous solution by activated alumina," Water, Air, and Soil Pollution, vol. 133, no. 1/4, pp. 349-361, 2002.

[14] E. Tchomgui-Kamga, V. Alonzo, C. P. Nanseu-Njiki, N. Audebrand, E. Ngameni, and A. Darchen, "Preparation and characterization of charcoals that contain dispersed aluminum oxide as adsorbents for removal of fluoride from drinking water," Carbon, vol. 48, no. 2, pp. 333-343, 2010.

[15] B. Grzmil and J. Wronkowski, "Removal of phosphates and fluorides from industrial wastewater," Desalination, vol. 189, no. 1-3, pp. 261-268, 2006.

[16] A. Dhillon, M. Nair, S. K. Bhargava, and D. Kumar, "Excellent fluoride decontamination and antibacterial efficacy of $\mathrm{Fe}-\mathrm{Ca}-$ Zr hybrid metal oxide nanomaterial," Journal of Colloid and Interface Science, vol. 457, pp. 289-297, 2015.

[17] F. Venditti, F. Cuomo, G. Giansalvo, M. Giustini, G. Cinelli, and F. Lopez, "Fluorides decontamination by means of aluminum polychloride based commercial coagulant," Journal of Water Process Engineering, vol. 26, pp. 182-186, 2018.

[18] M. Pontié, C. Diawara, A. Lhassani et al., "Chapter 2 Water defluoridation processes: a review. Application: nanofiltration (NF) for future large-scale pilot plants," Advances in Fluorine Science, vol. 2, pp. 49-80, 2006.

[19] H. Jin, Z. Ji, J. Yuan et al., "Research on removal of fluoride in aqueous solution by alumina-modified expanded graphite composite," Journal of Alloys and Compounds, vol. 620, pp. 361-367, 2015.

[20] N. Chen, C. Feng, and M. Li, "Fluoride removal on Fe-Alimpregnated granular ceramic adsorbent from aqueous solution," Clean Technologies and Environmental Policy, vol. 16, no. 3, pp. 609-617, 2014.

[21] K. Uma, G. T. Pan, and T. C. K. Yang, "The preparation of porous sol-gel silica with metal organic framework MIL-101(Cr) by microwave-assisted hydrothermal method for adsorption chillers," Materials, vol. 10, no. 6, p. 610, 2017.

[22] M. Eddaoudi, J. Kim, N. Rosi et al., "Systematic design of pore size and functionality in isoreticular MOFs and their application in methane storage," Science, vol. 295, no. 5554, pp. 469-472, 2002.

[23] H. Furukawa, K. E. Cordova, M. O'Keeffe, and O. M. Yaghi, "The chemistry and applications of metal-organic frameworks," Science, vol. 341, no. 6149, p. 974, 2013.

[24] F. Wang, W. Liu, S. J. Teat et al., "Chromophore-immobilized luminescent metal-organic frameworks as potential lighting phosphors and chemical sensors," Chemical Communications, vol. 52, no. 67, pp. 10249-10252, 2016.

[25] E. Haque, J. W. Jun, and S. H. Jhung, “Adsorptive removal of methyl orange and methylene blue from aqueous solution with a metal-organic framework material, iron terephthalate (MOF-235)," Journal of Hazardous Materials, vol. 185, no. 1, pp. 507-511, 2011.

[26] E. Adatoz and S. Keskin, "Application of MD simulations to predict membrane properties of MOFs," Journal of NanoMaterials, vol. 2015, Article ID 136867, 9 pages, 2015.

[27] S. Wang, C. M. McGuirk, A. d'Aquino, J. A. Mason, and C. A. Mirkin, "Metal-organic framework nanoparticles," Advanced Materials, vol. 30, no. 37, article 1800202, 2018.

[28] Z. Sumer and S. Keskin, "Computational screening of MOFbased mixed matrix membranes for $\mathrm{CO}_{2} / \mathrm{N}_{2}$ separations," Journal of NanoMaterials, vol. 2016, Article ID 6482628, 12 pages, 2016.

[29] M. Lismont, L. Dreesen, and S. Wuttke, "Metal-organic framework nanoparticles in photodynamic therapy: current status and perspectives," Advanced Functional Materials, vol. 12, no. 3, pp. 2094-2105, 2018.

[30] T. M. Al-Jadir and F. R. Siperstein, "The influence of the pore size in metal-organic frameworks in adsorption and separation of hydrogen sulphide: a molecular simulation study," Microporous and Mesoporous Materials, vol. 271, pp. 160168, 2018.

[31] S. Karmakar, J. Dechnik, C. Janiak, and S. De, "Aluminium fumarate metal-organic framework: a super adsorbent for fluoride from water," Journal of Hazardous Materials, vol. 303, pp. 10-20, 2016.

[32] X. Zhao, D. Liu, H. Huang, W. Zhang, Q. Yang, and C. Zhong, "The stability and defluoridation performance of MOFs in fluoride solutions," Microporous and Mesoporous Materials, vol. 185, pp. 72-78, 2014.

[33] Y. Xue, W. Sun, Q. Wang, L. Cao, and J. Yang, "Sparsely loaded Pt/MIL-96(Al) MOFs catalyst with enhanced activity for H2-SCR in a gas diffusion reactor under $80^{\circ} \mathrm{C}$," Chemical Engineering Journal, vol. 335, pp. 612-620, 2018.

[34] X. Yang, S. Deng, F. Peng, and T. Luo, "A new adsorbent of a Ce ion-implanted metal-organic framework (MIL-96) with high-efficiency $\mathrm{Ce}$ utilization for removing fluoride from 
water," Dalton Transactions, vol. 46, no. 6, pp. 1996-2006, 2017.

[35] T. Loiseau, L. Lecroq, C. Volkringer et al., "MIL-96, a porous aluminum trimesate 3D structure constructed from a hexagonal network of 18 -membered rings and $\mu 3$-oxo-centered trinuclear units," Journal of the American Chemical Society, vol. 128, no. 31, pp. 10223-10230, 2006.

[36] C. Zhang, Y. Li, T. J. Wang, Y. Jiang, and J. Fok, "Synthesis and properties of a high-capacity iron oxide adsorbent for fluoride removal from drinking water," Applied Surface Science, vol. 425, pp. 272-281, 2017.

[37] I. Langmuir, "The adsorption of gases on plane surfaces of glass, mica and platinum," Journal of Chemical Physics, vol. 40, no. 9, pp. 1361-1403, 1918.

[38] R. A. Anayurt, A. Sari, and M. Tuzen, "Equilibrium, thermodynamic and kinetic studies on biosorption of $\mathrm{Pb}$ (II) and Cd(II) from aqueous solution by macrofungus (Lactarius scrobiculatus) biomass," Chemical Engineering Journal, vol. 236, pp. 341-347, 2014.

[39] P. N. Dave and L. V. Chopda, "Application of iron oxide nanomaterials for the removal of heavy metals," Journal of Nanotechnology, vol. 2014, Article ID 398569, 14 pages, 2014.

[40] D. L. Childers, J. Corman, M. Edwards, and J. J. Elser, "Sustainability challenges of phosphorus and food: solutions from closing the human phosphorus cycle," Bioscience, vol. 61, no. 2, pp. 117-124, 2011.

[41] Y. L. Yan, M. A. Helfand, and C. R. Clayton, "Evaluation of the effect of surface roughness on thin film thickness measurements using variable angle XPS," Applied Surface Science, vol. 37, no. 4, pp. 395-405, 1989.

[42] Y. Yu, L. Yu, and J. Paul Chen, "Adsorption of fluoride by $\mathrm{Fe}-\mathrm{Mg}-\mathrm{La}$ triple-metal composite: adsorbent preparation, illustration of performance and study of mechanisms," Chemical Engineering Journal, vol. 262, pp. 839-846, 2015.

[43] L. Luo, K. Chen, Q. Liu et al., "Zinc(II) and cadmium(II) complexes with 1,3,5-benzenetricarboxylate and imidazolecontaining ligands: structural variation via reaction temperature and solvent," Crystal Growth and Design, vol. 13, no. 6, pp. 2312-2321, 2013.

[44] E. K. Putra, R. Pranowo, J. Sunarso, N. Indraswati, and S. Ismadji, "Performance of activated carbon and bentonite for adsorption of amoxicillin from wastewater: mechanisms, isotherms and kinetics," Water Research, vol. 43, no. 9, pp. 2419-2430, 2009.

[45] N. Kannan and M. M. Sundaram, "Kinetics and mechanism of removal of methylene blue by adsorption on various carbons-a comparative study," Dyes and Pigments, vol. 51, no. 1, pp. 25-40, 2001.

[46] C. Sairam Sundaram, N. Viswanathan, and S. Meenakshi, "Defluoridation of water using magnesia/chitosan composite," Journal of Hazardous Materials, vol. 163, no. 2-3, pp. 618-624, 2009.

[47] S. H. Dhawane, A. A. Khan, K. Singh, A. Tripathi, R. Hasda, and G. Halder, "Insight into optimization, isotherm, kinetics, and thermodynamics of fluoride adsorption onto activated alumina," Environmental Progress \& Sustainable Energy, vol. 37, no. 2, pp. 766-776, 2018.

[48] P. Liang, R. An, R. Li, and D. Wang, "Comparison of $\mathrm{La}^{3+}$ and mixed rare earths-loaded magnetic chitosan beads for fluoride adsorption," International Journal of Biological Macromolecules, vol. 111, pp. 255-263, 2018.
[49] S. M. Maliyekkal, A. K. Sharma, and L. Philip, "Manganeseoxide-coated alumina: a promising sorbent for defluoridation of water," Water Research, vol. 40, no. 19, pp. 3497-3506, 2006.

[50] A. Bansiwal, P. Pillewan, R. B. Biniwale, and S. S. Rayalu, "Copper oxide incorporated mesoporous alumina for defluoridation of drinking water," Microporous and Mesoporous Materials, vol. 129, no. 1-2, pp. 54-61, 2010.

[51] N. Zhang, X. Yang, X. Yu et al., “Al-1,3,5-benzenetricarboxylic metal-organic frameworks: a promising adsorbent for defluoridation of water with $\mathrm{pH}$ insensitivity and low aluminum residual," Chemical Engineering Journal, vol. 252, pp. 220229, 2014. 


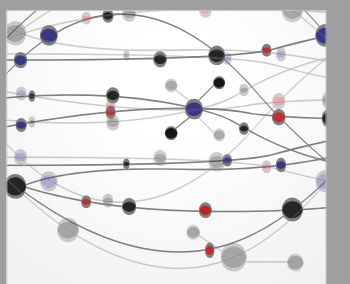

The Scientific World Journal
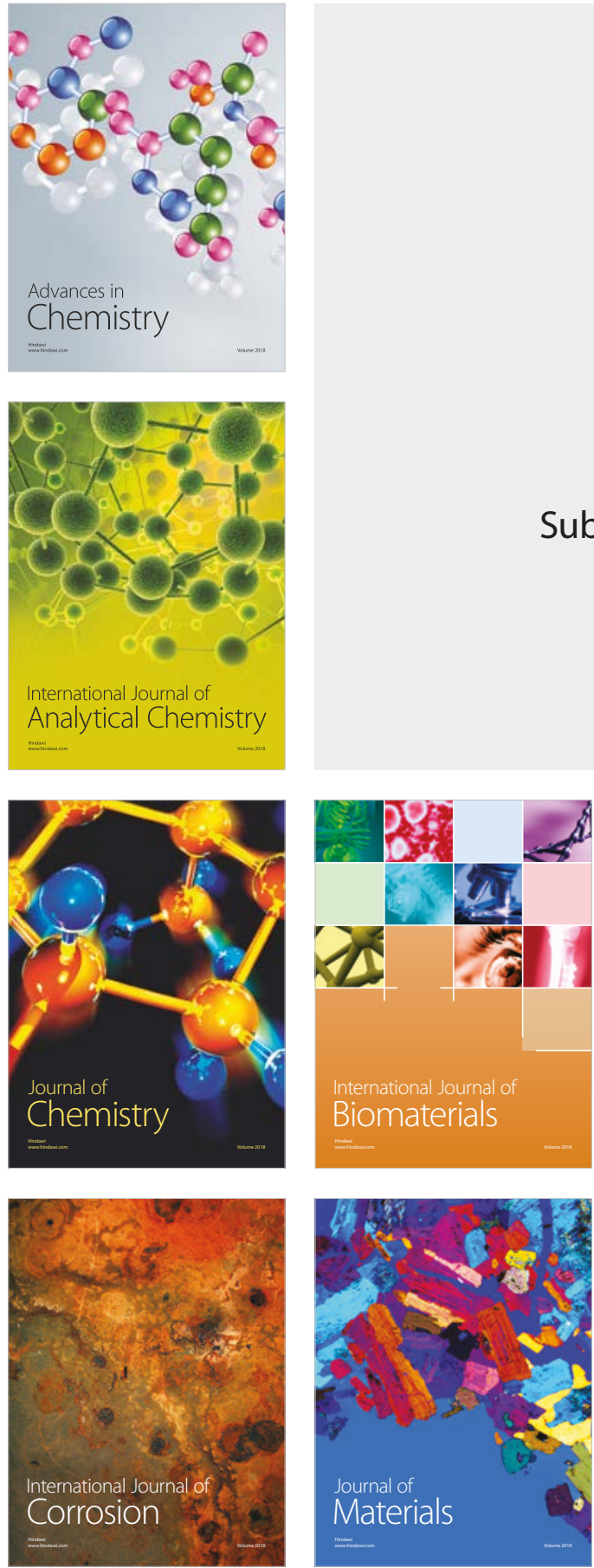

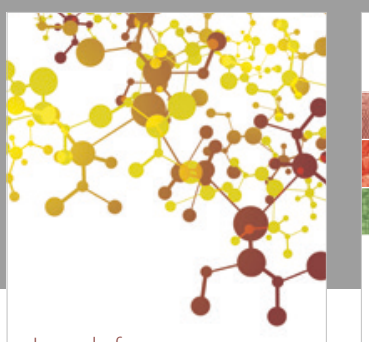

Journal of

Applied Chemistry
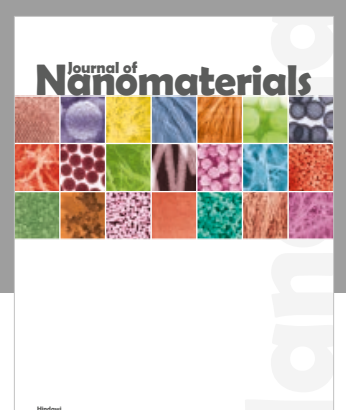

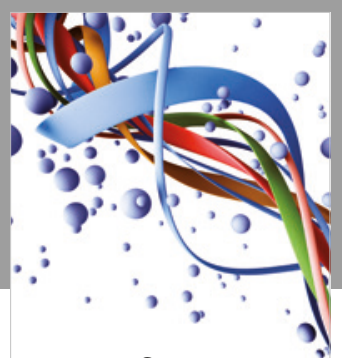

Scientifica

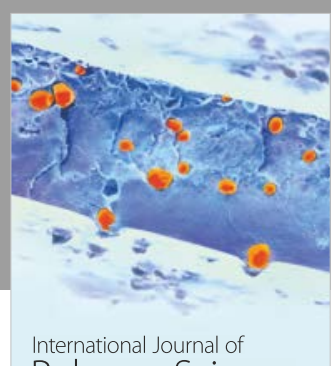

Polymer Science

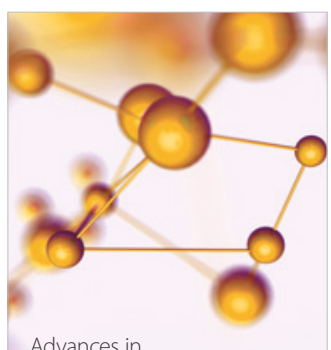

Physical Chemistry
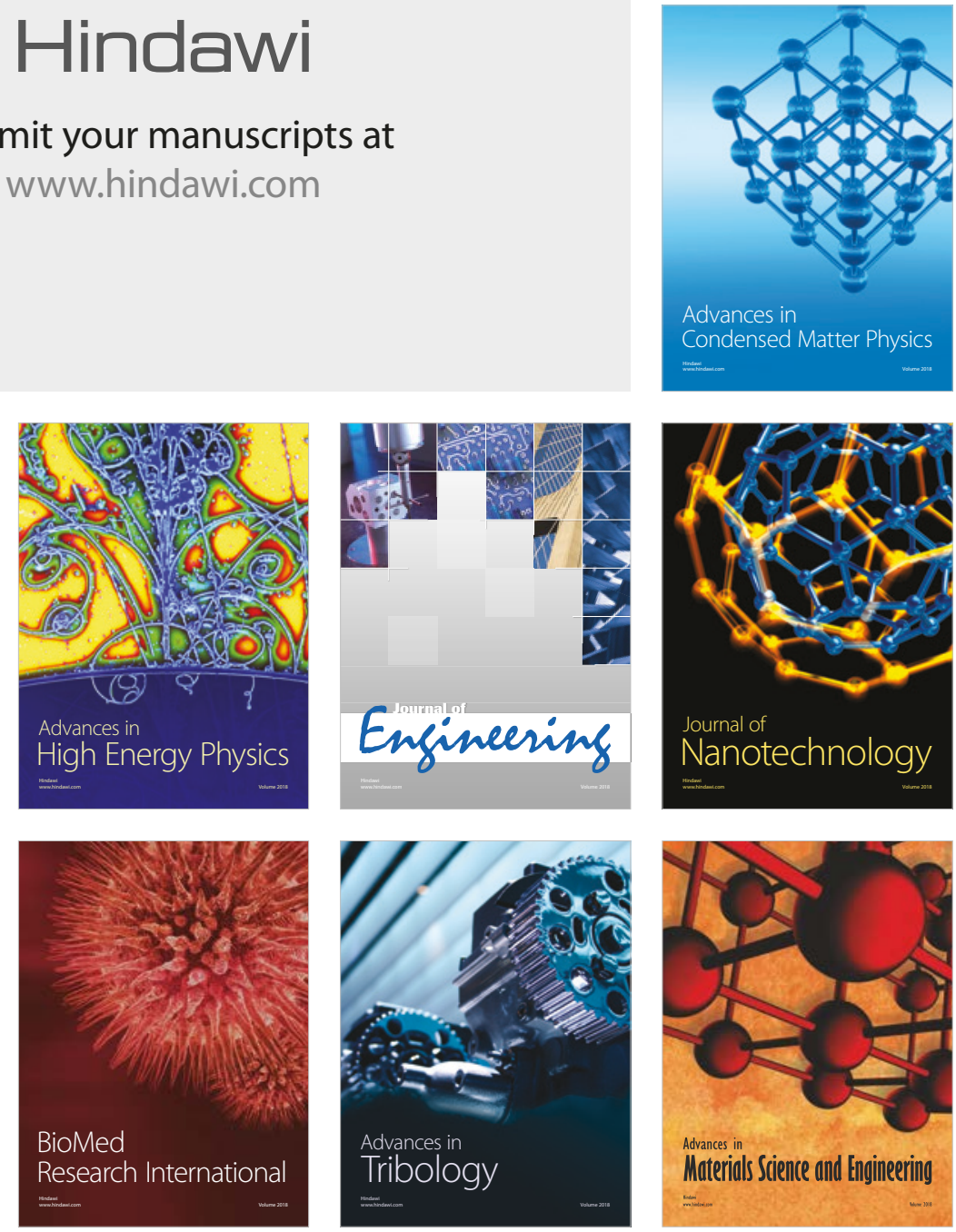